\title{
Contemporary relevance of TID: Based on the company it keeps
}

\author{
Jamieson $M$. Bourque, $M D, M H S^{a, b}$ \\ ${ }^{a}$ Cardiovascular Division and the Cardiovascular Imaging Center, Department of Medicine, \\ University of Virginia Health System, Charlottesville, VA \\ ${ }^{\mathrm{b}}$ Department of Radiology, University of Virginia Health System, Charlottesville, VA
}

Received Mar 11, 2015; accepted Mar 11, 2015

doi: $10.1007 / \mathrm{s} 12350-015-0122-7$

\section{See related article, pp. 526-534}

A fundamental strength of SPECT myocardial perfusion imaging (MPI) has been the low event rate in most patients with a negative scan, as low as $0.6 \% \cdot$ year $^{-1}$ in those undergoing exercise stress. ${ }^{1}$ However, the rate is higher in other populations such as those undergoing vasodilator stress $\left(1.8 \% \cdot\right.$ year $\left.^{-1}\right)$, in part due to the occurrence of balanced ischemia, in which patients with multivessel ischemia have normal relative perfusion patterns. ${ }^{2}$ Multiple ancillary variables assist in detecting high-risk patients with normal perfusion scans, including exercise workload, ischemic STchanges, and functional abnormalities on gatedSPECT. ${ }^{3,4}$ One such marker, transient ischemic dilation (TID), is well documented to provide incremental diagnostic and prognostic value, although there is controversy regarding its appropriate application across varying clinical situations.

TID is hypothesized to result from two primary pathophysiologic mechanisms, reduced function with resultant elevated end-systolic volume and diffuse subendocardial hypoperfusion. There are studies that support both hypotheses, and the true mechanism is likely a combination of both. ${ }^{5,6}$ TID has been validated across multiple varying clinical parameters that impact the optimal abnormal threshold. Thresholds for TID have been identified in 1- and 2-day protocols using multiple tracers, including $\mathrm{Tl}-201,{ }^{7} \mathrm{Tc}-99 \mathrm{~m},{ }^{8}$ dual-

\footnotetext{
Reprint requests: Jamieson M. Bourque, MD, MHS, Cardiovascular Division and the Cardiovascular Imaging Center, Department of Medicine, University of Virginia Health System, Box 800158, 1215 Lee Street, Charlottesville, VA 22908; jbourque@virginia.edu J Nucl Cardiol 2015;22:535-8.

1071-3581/\$34.00

Copyright (C) 2015 American Society of Nuclear Cardiology.
}

isotope protocols, ${ }^{9}$ and even $\mathrm{Rb}-82$ positron emission tomography (PET). ${ }^{10}$ There are data in exercise stress ${ }^{7,9}$ and with multiple pharmacologic agents, including adenosine,${ }^{11}$ dipyridamole, ${ }^{12}$ and regadenoson ${ }^{13}$ in the setting of dual-isotope SPECT. In this issue of the Journal, Golzar et al provide the first data establishing the abnormal TID threshold in patients undergoing reststress Tc-99m SPECT with regadenoson stress. They identified an optimal threshold of 1.31 for TID using this set of clinical parameters in a cohort of 100 patients with a low likelihood of coronary artery disease (CAD). However, they found TID to lack incremental diagnostic value in a cohort of 547 patients with coronary angiography within 6 months of SPECT imaging, adding to the controversy regarding the significance of TID. It is likely that the relevance of TID rests in the company it keeps.

\section{TID IN THE SETTING OF ABNORMAL MPI}

There are extensive data showing the diagnostic and prognostic value of TID in the setting of abnormal MPI. Early studies using Tl-201 by Chouraqui et $\mathrm{al}^{14}$ and Takeishi et $\mathrm{al}^{15}$ did not specifically examine the subgroup with perfusion abnormalities but found a close association between TID and severe and extensive CAD in populations with a high incidence of MPI abnormalities. Mazzanti et al specifically examined a population with MPI abnormalities on exercise dualisotope Tc-99m/Tl-201 SPECT. ${ }^{9}$ They found a close association between TID and perfusion abnormalities. No patients with a SSS $<4$ had TID. Moreover, the presence of both an abnormal MPI and TID was associated with $71 \%$ sensitivity and $95 \%$ specificity for severe and extensive CAD. Abidov et al showed a $65 \%$ rate of severe and extensive CAD in the 84 patients with an abnormal TID ratio and $\geq 5 \%$ left ventricular (LV) ischemia. ${ }^{11}$ TID has been shown to predict events 
in a population with fixed perfusion defects and a low left ventricular ejection fraction (LVEF). A contemporary analysis by $\mathrm{Xu}$ et al showed a higher incidence of TID and an increased sensitivity for severe CAD in those with perfusion abnormalities. ${ }^{16}$

\section{EARLY LITERATURE ON TID IN OTHERWISE NORMAL MPI}

In order to improve the diagnostic accuracy of SPECT MPI and reduce undetected balanced ischemia, the next logical step was the application of TID in patients with an otherwise normal MPI study. However, in contrast to the consistent data showing the incremental value of TID in the setting of an abnormal MPI, the role of TID in patients with an otherwise normal MPI has been more controversial.

Early studies examining the normal MPI population showed a more robust benefit of TID. One approach has been to examine the presence of TID in patients with confirmed severe and extensive CAD. Weiss et al performed one of the first studies examining TID in 89 patients who underwent planar Tl-201 and coronary angiography, and found multivessel or left main disease in all seven subjects with TID and no reversible defects. ${ }^{7}$ However, $71 \%$ had evidence of a prior myocardial infarction (MI), and their MPI technique had limited sensitivity. In the cohort of 356 studied by Abidov et al, 3 of the 11 patients with TID but otherwise normal MPI had severe and extensive CAD $(27.3 \%)$ vs only $1 / 79$ $(1.3 \%)$ with no TID or MPI abnormalities. ${ }^{11}$ A limitation of these analyses is that they examined a highly selected population, significantly increasing the risk of referral bias.

A second method has been to compare the event rate of patients with and without TID in the more generalized population undergoing MPI with or without subsequent angiography. Abidov et al examined two populations without significant MPI abnormalities. ${ }^{17}$ In the first population of 1,560 subjects with a SSS of 0 and no resting $\mathrm{LV}$ enlargement, TID increased the risk of cardiac events threefold $\left(2.4 \%\right.$ vs $0.8 \%$.year ${ }^{-1}$, $P=.001)$. The increased risk with TID was present but attenuated in a second population of 2,037 subjects without significant perfusion defects, defined as a $\mathrm{SSS}<3\left(2.2 \%\right.$ vs $1.0 \% \cdot$ year $\left.^{-1}, P=.002\right)$.

\section{CONTEMPORARY STUDIES ON TID IN OTHERWISE NORMAL MPI}

In contrast, more contemporary studies examining the normal MPI population have not found TID to provide incremental value in the identification of severe and extensive CAD. A 9-year retrospective analysis published in this Journal in 2011 by Valdiziezo et al found no increase in the presence of left main or multivessel disease in the 28 patients with TID (11\%) vs 68 without $(16 \%)$. They also found no difference in the incidence of any CAD detected on invasive coronary angiography. Halligan et al confirmed the absence of an increase in multivessel disease in patients with TID and extended the results to include no increase in calcium score or extent of atherosclerosis as measured by cardiac CT in 1,553 consecutive patients who underwent a SPECT MPI and cardiac CT within 1 month between 2006 and 2011. ${ }^{18}$ The incidence of MACE was also similar. There is potential selection bias in this cohort, however, as the authors could not control for parameters that drove the decision to perform cardiac CT rather than invasive coronary angiography.

Mandour Ali et al suggest that measurement variance is the cause of TID in patients with normal SPECT MPI. They supported this hypothesis by showing a low incidence at the 2 standard deviation threshold in 688 subjects imaged in 2010, yielding a predictive accuracy of $26 \%{ }^{19}$

In this issue of the Journal, Golzar et al studied 547 subjects, including $219(40 \%)$ with a SSS $<4$, and found no significant difference in the prevalence of severe CAD by TID status ( $8.1 \%$ for TID+ vs $9.8 \%$ for TID-, $P=.74)$. ROC analysis found TID to have poor discriminatory capacity for severe and extensive CAD (AUC $=0.55, P=.25$ ). Their conclusions were supported by a robust sensitivity analysis that varied the definition of severe CAD and the TID threshold with an unchanged final result. A stepwise multivariable logistic regression analysis found that TID did not provide incremental diagnostic value for the identification of severe CAD over clinical predictors or the summed stress score (SSS).

As suggested by Golzar et al, one possible reason for the lack of incremental benefit of TID in contemporary analyses is the decreasing prevalence of ischemia on SPECT MPI studies. The prevalence of ischemic SPECT studies has decreased from $29.6 \%$ in 1991 to $5.0 \%$ in 2009 , a $>$ fivefold decrease. ${ }^{20}$ As the presence and severity of CAD decrease, the positive predictive value of TID for severe CAD declines, and this finding is more likely to represent an alternative such as a manifestation of cardiomyopathy or measurement variance. $^{19}$

This hypothesis is supported by a 2013 analysis by Doukky et al, in which TID was predictive in a subgroup of 294 patients with diabetes or known CAD and normal MPI defined as SSS $=0$, normal LV volumes, and

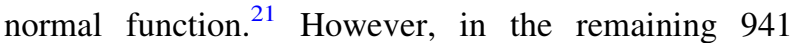
subjects without DM or CAD, TID was not predictive of cardiac death or nonfatal MI. Known CAD, DM, and 
chronic kidney disease are all significant comorbidities that increase the likelihood that the presence of TID is a true positive. ${ }^{22}$ Patients with DM are known to have a minimal warranty period after a normal MPI study. ${ }^{23}$ The question remains whether the predictive power of TID in those with risk factors such as DM is due to the presence of severe and extensive CAD at the time of testing, or whether TID is the manifestation of another process with an adverse prognostic effect.

\section{TID CLINICAL APPLICATION}

An algorithm for the clinical application of TID is proposed in Figure 1. Multiple studies suggest that TID in the setting of abnormal MPI is high risk, and further evaluation of coronary anatomy is warranted.9,11,14-16 Normal MPI studies with SSS $<4$ and normal LV function and systolic volumes have a low likelihood of obstructive CAD and a low subsequent event rate in the absence of high-risk comorbidities. Given that recent studies show no incremental benefit of TID in this population, these patients can be observed with careful follow-up. ${ }^{18,24}$ Patients with high-risk comorbidities such as known CAD, diabetes mellitus, or chronic kidney disease have an intermediate risk, and TID may have benefit in this subgroup. ${ }^{21}$ However, the majority of these patients will not have obstructive disease on angiography. Accordingly, calcium scoring could be used as an additional risk stratification method to reduce unnecessary invasive testing. Patients with a calcium score of $<400$ have a low risk of severe and extensive CAD and

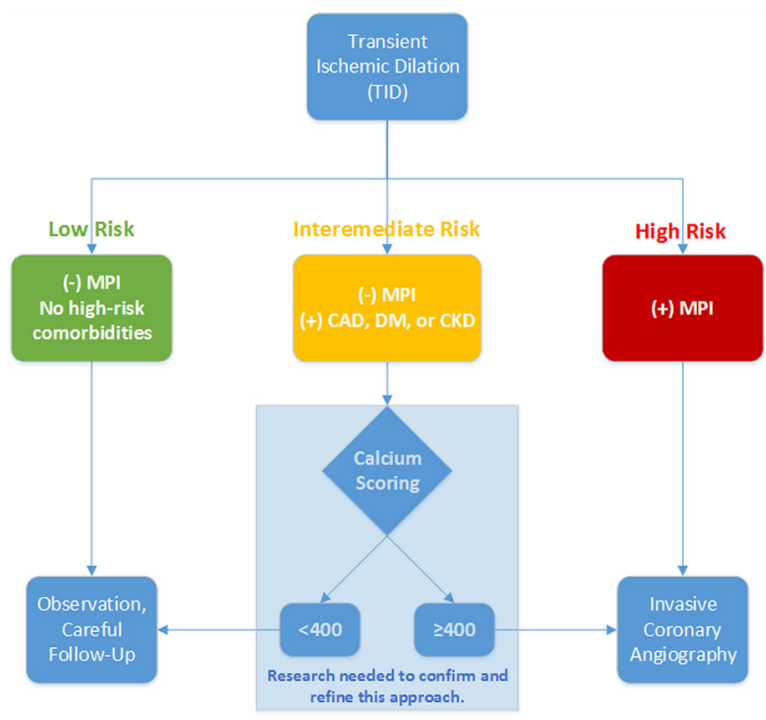

Figure 1. Proposed algorithm for the clinical application of transient ischemic dilation (TID). $C A D$, Coronary artery disease; $C K D$, chronic kidney disease; $D M$, diabetes mellitus; $M P I$, myocardial perfusion imaging. subsequent cardiac events and could be observed. In contrast, those with a score $\geq 400$ are higher risk and a coronary evaluation could be considered. The higher risk of these comorbidities and use of calcium scoring in this fashion have not been validated and should be confirmed and refined through clinical research.

\section{RESEARCH CHALLENGES AND FUTURE DIRECTIONS}

With respect to the identification of severe and extensive CAD, the populations assessed in the TID literature have been cohorts undergoing both MPI and coronary angiography, a highly selective cohort. The highly specialized nature of this cohort increases the risk of selection bias. To reduce this bias, research in the general MPI population has been performed, but has looked only at events. Additional research should examine the prevalence of proximal vessel or multivessel obstructive CAD in this general population. This could be performed with low risk through CT coronary angiography given the newer low-dose protocols employed. ${ }^{25}$

Use of a broad MPI population with anatomic correlation could allow more robust identification of which comorbidities, if any, obligate additional risk stratification. Additional future directions include the refinement of TID cutoffs across a variety of hardware and software protocols, the transition to routine quantitative measurement, and the development of practices that minimize current TID assessment limitations such as improper slice alignment, motion artifact, and normal variance in small ventricles. ${ }^{9,26,27}$ In this contemporary era of varying clinical relevance of TID, refined risk profiles could allow for improved identification of patients with severe and extensive CAD without exposing those at lower risk to the hazards of unnecessary testing.

\section{Acknowledgments}

Dr. Bourque receives research grant support from Astellas Pharma.

\section{References}

1. Klocke FJ, Baird MG, Lorell BH, Bateman TM, Messer JV, Berman DS, et al. ACC/AHA/ASNC guidelines for the clinical use of cardiac radionuclide imaging-Executive summary: A report of the American College of Cardiology/American Heart Association Task Force on Practice Guidelines (ACC/AHA/ASNC Committee to Revise the 1995 Guidelines for the Clinical Use of Cardiac Radionuclide Imaging). J Am Coll Cardiol 2003;42:1318-33.

2. Navare SM, Mather JF, Shaw LJ, Fowler MS, Heller GV. Comparison of risk stratification with pharmacologic and exercise stress myocardial perfusion imaging: A meta-analysis. J Nucl Cardiol 2004;11:551-61. 
3. Weiner DA, Ryan TJ, McCabe CH, Chaitman BR, Sheffield LT, Ferguson JC, et al. Prognostic importance of a clinical profile and exercise test in medically treated patients with coronary artery disease. J Am Coll Cardiol 1984;3:772-9.

4. Abidov A, Germano G, Hachamovitch R, Berman DS. Gated SPECT in assessment of regional and global left ventricular function: Major tool of modern nuclear imaging. J Nucl Cardiol 2006; 13:261-79.

5. Marcassa C, Galli M, Baroffio C, Campini R, Giannuzzi P. Transient left ventricular dilation at quantitative stress-rest sestamibi tomography: Clinical, electrocardiographic, and angiographic correlates. J Nucl Cardiol 1999;6:397-405.

6. Bestetti A, Di Leo C, Alessi A, Triulzi A, Tagliabue L, Tarolo GL. Post-stress end-systolic left ventricular dilation: A marker of endocardial post-ischemic stunning. Nucl Med Commun 2001;22: 685-93.

7. Weiss AT, Berman DS, Lew AS, Nielsen J, Potkin B, Swan HJ, et al. Transient ischemic dilation of the left ventricle on stress thallium-201 scintigraphy: A marker of severe and extensive coronary artery disease. J Am Coll Cardiol 1987;9:752-9.

8. Peace RA, McKiddie FI, Staff RT, Gemmell HG. Comparison of methods for quantification of transient ischaemic dilation in myocardial perfusion SPET. Nucl Med Commun 2000;21:971-6.

9. Mazzanti M, Germano G, Kiat H, Kavanagh PB, Alexanderson E, Friedman JD, et al. Identification of severe and extensive coronary artery disease by automatic measurement of transient ischemic dilation of the left ventricle in dual-isotope myocardial perfusion SPECT. J Am Coll Cardiol 1996;27:1612-20.

10. Rischpler C, Higuchi T, Fukushima K, Javadi MS, Merrill J, Nekolla SG, et al. Transient ischemic dilation ratio in $82 \mathrm{Rb}$ PET myocardial perfusion imaging: Normal values and significance as a diagnostic and prognostic marker. J Nucl Med 2012;53:723-30.

11. Abidov A, Bax JJ, Hayes SW, Cohen I, Nishina H, Yoda S, et al. Integration of automatically measured transient ischemic dilation ratio into interpretation of adenosine stress myocardial perfusion SPECT for detection of severe and extensive CAD. J Nucl Med 2004;45:1999-2007.

12. Hung GU, Lee KW, Chen CP, Lin WY, Yang KT. Relationship of transient ischemic dilation in dipyridamole myocardial perfusion imaging and stress-induced changes of functional parameters evaluated by Tl-201 gated SPECT. J Nucl Cardiol 2005;12:26875 .

13. Katz JS, Ruisi M, Giedd KN, Rachko M. Assessment of transient ischemic dilation (TID) ratio in gated SPECT myocardial perfusion imaging (MPI) using regadenoson, a new agent for pharmacologic stress testing. J Nucl Cardiol 2012;19:727-34.

14. Chouraqui P, Rodrigues EA, Berman DS, Maddahi J. Significance of dipyridamole-induced transient dilation of the left ventricle during thallium-201 scintigraphy in suspected coronary artery disease. Am J Cardiol 1990;66:689-94.

15. Takeishi Y, Chiba J, Abe S, Komatani A, Takahashi K, Tomoike H. Noninvasive identification of left main and three-vessel coronary artery disease by thallium-201 single photon emission computed tomography during adenosine infusion. Ann Nucl Med 1994;8:1-7.

16. Xu Y, Arsanjani R, Clond M, Hyun M, Lemley M Jr, Fish M, et al. Transient ischemic dilation for coronary artery disease in quantitative analysis of same-day sestamibi myocardial perfusion SPECT. J Nucl Cardiol 2012;19:465-73.

17. Abidov A, Bax JJ, Hayes SW, Hachamovitch R, Cohen I, Gerlach $\mathrm{J}$, et al. Transient ischemic dilation ratio of the left ventricle is a significant predictor of future cardiac events in patients with otherwise normal myocardial perfusion SPECT. J Am Coll Cardiol 2003;42:1818-25.

18. Halligan WT, Morris PB, Schoepf UJ, Mischen BT, Spearman JV, Spears JR, et al. Transient ischemic dilation of the left ventricle on SPECT: Correlation with findings at coronary CT angiography. J Nucl Med 2014;55:917-22.

19. Mandour Ali MA. Bourque JM, Allam AH, Beller GA, Watson DD. The prevalence and predictive accuracy of quantitatively defined transient ischemic dilation of the left ventricle on otherwise normal SPECT myocardial perfusion imaging studies. J Nucl Cardiol 2011;18:1036-43.

20. Rozanski A, Gransar H, Hayes SW, Min J, Friedman JD, Thomson LE, et al. Temporal trends in the frequency of inducible myocardial ischemia during cardiac stress testing: 1991 to 2009. J Am Coll Cardiol 2013;61:1054-65.

21. Doukky R, Frogge N, Bayissa YA, Balakrishnan G, Skelton JM, Confer $\mathrm{K}$, et al. The prognostic value of transient ischemic dilatation with otherwise normal SPECT myocardial perfusion imaging: A cautionary note in patients with diabetes and coronary artery disease. J Nucl Cardiol 2013;20:774-84.

22. Bourque JM, Beller GA. Stress myocardial perfusion imaging for assessing prognosis: An update. JACC Cardiovasc Imaging 2011;4:1305-19.

23. Bourque JM, Patel CA, Ali MM, Perez M, Watson DD, Beller GA. Prevalence and predictors of ischemia and outcomes in outpatients with diabetes mellitus referred for single-photon emission computed tomography myocardial perfusion imaging. Circ Cardiovasc Imaging 2013;6:466-77.

24. Valdiviezo C, Motivala AA, Hachamovitch R, Chamarthy M, Navarro PC, Ostfeld RJ, et al. The significance of transient ischemic dilation in the setting of otherwise normal SPECT radionuclide myocardial perfusion images. J Nucl Cardiol 2011;18:220-9.

25. Raff GL. Radiation dose from coronary CT angiography: Five years of progress. J Cardiovasc Comput Tomogr 2010;4:365-74.

26. Hansen CL, Sangrigoli R, Nkadi E, Kramer M. Comparison of pulmonary uptake with transient cavity dilation after exercise thallium-201 perfusion imaging. J Am Coll Cardiol 1999;33:13237.

27. Fitzgerald J, Danias PG. Effect of motion on cardiac SPECT imaging: Recognition and motion correction. J Nucl Cardiol 2001;8:701-6. 